\title{
Evidence of surface loss as ubiquitous limiting damping mechanism in SiN micro- and nanomechanical resonators
}

\author{
Villanueva, Luis Guillermo; Schmid, Silvan
}

Published in:

Physical Review Letters

Link to article, DOI:

10.1103/PhysRevLett.113.227201

Publication date:

2014

Document Version

Publisher's PDF, also known as Version of record

Link back to DTU Orbit

Citation (APA):

Villanueva, L. G., \& Schmid, S. (2014). Evidence of surface loss as ubiquitous limiting damping mechanism in SiN micro- and nanomechanical resonators. Physical Review Letters, 113, 227201.

https://doi.org/10.1103/PhysRevLett.113.227201

\section{General rights}

Copyright and moral rights for the publications made accessible in the public portal are retained by the authors and/or other copyright owners and it is a condition of accessing publications that users recognise and abide by the legal requirements associated with these rights.

- Users may download and print one copy of any publication from the public portal for the purpose of private study or research.

- You may not further distribute the material or use it for any profit-making activity or commercial gain

- You may freely distribute the URL identifying the publication in the public portal 


\title{
Evidence of Surface Loss as Ubiquitous Limiting Damping Mechanism in SiN Micro- and Nanomechanical Resonators
}

\author{
L. G. Villanueva ${ }^{1}$ and S. Schmid ${ }^{2, *}$ \\ ${ }^{1}$ Advanced NEMS Group, École Polytechnique Fédérale de Lausanne (EPFL), CH-1015 Lausanne, Switzerland \\ ${ }^{2}$ Department of Micro-and Nanotechnology, Technical University of Denmark, DTU Nanotech, DK-2800 Kongens Lyngby, Denmark \\ (Received 26 June 2014; published 25 November 2014)
}

\begin{abstract}
Silicon nitride ( $\mathrm{SiN}$ ) micro- and nanomechanical resonators have attracted a lot of attention in various research fields due to their exceptionally high quality factors $(Q s)$. Despite their popularity, the origin of the limiting loss mechanisms in these structures has remained controversial. In this Letter we propose an analytical model combining acoustic radiation loss with intrinsic loss. The model accurately predicts the resulting mode-dependent $Q$ s of low-stress silicon-rich and high-stress stoichiometric SiN membranes. The large acoustic mismatch of the low-stress membrane to the substrate seems to minimize radiation loss and $Q$ s of higher modes $(n \wedge m \geq 3)$ are limited by intrinsic losses. The study of these intrinsic losses in low-stress membranes reveals a linear dependence with the membrane thickness. This finding was confirmed by comparing the intrinsic dissipation of arbitrary (membranes, strings, and cantilevers) SiN resonators extracted from literature, suggesting surface loss as ubiquitous damping mechanism in thin SiN resonators with $Q_{\text {surf }}=\beta h$ and $\beta=6 \times 10^{10} \pm 4 \times 10^{10} \mathrm{~m}^{-1}$. Based on the intrinsic loss the maximal achievable $Q$ s and $Q f$ products for $\mathrm{SiN}$ membranes and strings are outlined.
\end{abstract}

DOI: 10.1103/PhysRevLett.113.227201

PACS numbers: 85.85.+j, 42.50.Wk, 42.60.Da

Since the discovery of the exceptionally high quality factors $(Q)$ of nanomechanical silicon nitride $(\mathrm{SiN})$ resonators [1,2], SiN strings and membranes have become the centerpiece of many experiments in the fields of cavity optomechanics [3-14] and sensor technology [15-20]. For example, in cavity optomechanics a high $Q$ at high frequencies is required in order to advance towards the quantum regime of the mechanical resonators, and in resonant sensors a high $Q$ enables a better resolution. Despite the continuous effort to understand and optimize $Q$ of $\mathrm{SiN}$ resonators, the underlying source of the limiting mechanism has remained controversial. On the one hand, it has been suggested by several groups that $\mathrm{SiN}$ resonators are limited by intrinsic losses [21-23]. Conversely, it has recently been suggested that radiation loss is the limiting factor for $Q$ in $\mathrm{SiN}$ membranes [24]. In this Letter we show that a model which combines intrinsic and acoustic radiation losses accurately predicts the mode-dependent $Q$ s of low- and high-stress $\mathrm{SiN}$ membranes. Finally, we show that the intrinsic loss in thin arbitrary SiN resonators scales with thickness. This is evidence that surface loss is the ubiquitous limiting damping mechanism in micro- and nanomechanical SiN resonators.

The exceptionally high $Q \mathrm{~s}$ of $\mathrm{SiN}$ resonators originate from the high intrinsic tensile stress $\sigma$ which increases the stored energy without significantly increasing the energy loss during vibration $[21,22,25]$. Assuming the energy loss to be coupled to the local out-of-plane bending during vibration, the intrinsic quality factor of a square membrane under tensile stress $Q_{\mathrm{intr}, \sigma}$ is given by [25]

$$
Q_{\mathrm{intr}, \sigma} \approx Q_{\mathrm{intr}}\left[2 \lambda+\left(n^{2}+m^{2}\right) \pi^{2} \lambda^{2}\right]^{-1},
$$

with $\lambda=(h / L) \sqrt{E /(12 \sigma)}$ where $Q_{\text {intr }}$ is the intrinsic quality factor of the relaxed resonator without the tensile stress (like, for example, a cantilever), $n, m$ are the mode numbers, $E$ the Young's modulus, $h$ the thickness, $L$ is the side length, and $\sigma$ is the tensile stress. The expression for strings can also be developed and the final result is (1) with $m=0$ and $n$ as the mode number, which is equal to an earlier model for $Q$ of loaded wires [26]. The value in square brackets in (1) is a $Q$-enhancing factor that comprises two terms. The left term is independent of the mode number and comes from the local curvature of the resonator at the clamped ends. The right term is dependent on the mode numbers and originates from the curvature of the antinodes. As per definition of a string or membrane $\lambda \ll 1$ [27]. Hence, the left term is a lot larger, that is, the damping due to the membrane curvature at the clamped ends usually dominates $Q_{\text {intr }, \sigma}$. The local bending at the clamping is decreasing exponentially with a decay length $L_{c}=L \lambda[25,26,28]$. For stoichiometric $\operatorname{SiN} L_{c} \approx 5 \times h$ and the peak intrinsic damping for a $30 \mathrm{~nm}$ thick resonator thus happens within a $150 \mathrm{~nm}$ wide band at the resonator ends close to the clamping.

Besides the intrinsic energy loss, the resonators can lose energy through phonons tunneling into the substrate, so-called acoustic radiation loss. It has been suggested that acoustic radiation loss in $\mathrm{SiN}$ membranes is strongly mode dependent and that modes with low mode numbers typically are limited by radiation loss [29]. An analytical model based on the coupling of membrane modes to free modes of the substrate has been fully developed $[29,30]$. For the sake of simplicity, we provide here the asymptotic limit for a square membrane 


$$
Q_{\mathrm{rad}} \approx 1.5 \alpha \frac{\rho_{s}}{\rho_{r}} \eta^{3} \frac{n^{2} m^{2}}{\left(n^{2}+m^{2}\right)^{3 / 2}} \frac{L}{h}
$$

with the "acoustic mismatch" (phase velocity ratio) between a semi-infinite substrate and the resonator $\eta \approx \sqrt{\left(E_{s} / \sigma\right)\left(\rho_{r} / \rho_{s}\right)}$ with the mass densities $\rho_{s}$ and $\rho_{r}$ of the substrate and resonator, respectively, and the Young's modulus of the substrate $E_{s}$. The prefactor $\alpha$ is a fitting parameter correcting for substrate imperfections resulting from the specific chip mounting conditions. Under ideal conditions of a semi-infinite substrate $\alpha=1$. Equation (2) is valid under the condition $n, m \gg \sqrt{n^{2}+m^{2}} / \eta$. Typically, $\eta \gg 1$ for SiN membranes and thus the radiation loss model is valid for all $n \sim m$. Destructive interference of the waves radiating into the substrate can lead to a suppression of the acoustic radiation loss for increasing harmonic modes $(n=m)$ [29]. From (2) it can be seen that acoustic radiation loss is minimal for harmonic modes $n=m$ and the envelope of maximal values is increasing linearly with the mode numbers $Q_{\mathrm{rad}} \propto n$. For strings, $Q_{\text {rad }} \propto L / w$ is predicted to be a function of the string width $w$ [31]. This effect has been observed with SiN strings where $Q$ increased with decreasing width and approached an asymptotic limit given by intrinsic losses [21].

According to (2), $Q_{\text {rad }}$ is a function of the acoustic mismatch $\eta$ between the resonator and a semi-infinite substrate. This has recently been demonstrated with nanomechanical SiN strings whose $Q$ s deteriorate when the acoustic mismatch is reduced by touching the anchor area with an AFM tip [32]. It has repeatedly been shown that especially lower mode $Q$ s are sensitive to the chip mounting conditions and that these $Q$ s can be increased by minimizing the contact between chip and support [7,21,24,33]. A successful way of suppressing radiation losses is to locate the mechanical structure within a well-designed phononic band gap structure. This removes the free frame modes around the membrane and suppresses the probability of phonon tunneling, i.e., radiation loss [34,35]. The measured maximal $Q$ s of modes with negligible radiation loss of such a $\mathrm{SiN}$ membrane had maximal $Q$ values that correspond to expected values obtained with similar membranes without the phononic band gap. This is strong evidence that $Q \mathrm{~s}$ in $\mathrm{SiN}$ membranes ultimately can be limited by intrinsic losses if the chip is mounted carefully. This notion is supported by the comparison of specific examples of $Q$ values of stoichiometric SiN strings [22] and membranes [24] from literature to the intrinsic (1) and acoustic radiation loss (2) models (see Supplemental Material [36]). It suggests that intrinsic loss is limiting $Q$ in both cases, which contradicts the conclusions made in [24] that radiation loss is the limiting mechanism.

There are strong indications that the overall mode dependence of $Q$ is best described by a combination of both models. In order to test this we compare low-stress silicon-rich $\mathrm{SiN}$ (SR-SiN) (from Norcada) and high-stress stoichiometric $\mathrm{SiN}$ (fabricated in-house) membrane $Q$ data to a combined model that takes into account both intrinsic and acoustic radiation losses

$$
Q^{-1}=Q_{\text {intr }, \sigma}^{-1}+Q_{\text {rad }}^{-1} .
$$

The membranes were characterized in the frequency domain with a lock-in amplifier (Zurich Instruments HF2PLL) in high vacuum (pressure $<10^{-5}$ mbar) at room temperature. The membrane motion was actuated in the linear regime with a piezoelectric shaker and detected with a laser vibrometer (MSA-500 Polytec $\mathrm{GmbH}$ ).

Figures 1(a) and 1(b) show the measured $Q$ s for various modes of a SR-SiN and stoichiometric SiN membrane, respectively, with equal dimensions. The combined model (3), based on the exact solution of the radiation loss model [30], predicts the measured values of both membranes with good accuracy for a single chosen set of parameters $Q_{\text {intr }}$ and $\alpha$. These parameters resulted in the best possible fit for both membranes. All the modes in Fig. 1 fulfill the conditions required for the validity of the radiation loss model. The maximal $Q$ s of the low-stress membrane Fig. 1(a) produce an envelope of maximal $Q$ values which
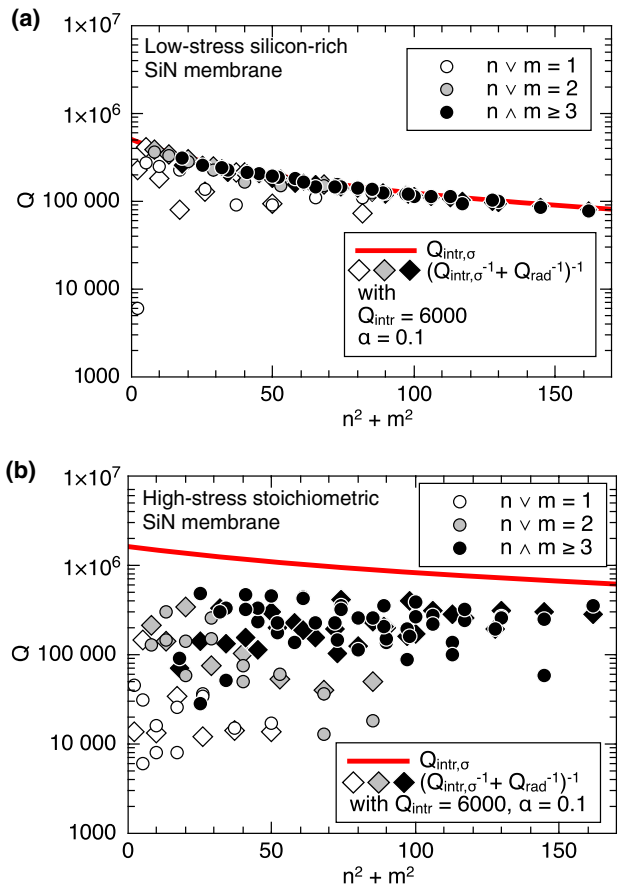

FIG. 1 (color online). $Q$ s for modes $(n \wedge m \leq 9)$ of a (a) square SR-SiN membrane $(L \approx 250 \mu \mathrm{m}, \sigma=92 \mathrm{MPa}, h \approx 100 \mathrm{~nm})$ and (b) square stoichiometric $\operatorname{SiN}$ membrane $(L \approx 250 \mu \mathrm{m}$, $\sigma=988 \mathrm{MPa}, h \approx 100 \mathrm{~nm})$. The chips were fixed with a double sticky carbon disc (Agar Scientific). The red line represents the highest quality factor value envelope due to intrinsic loss (1). The diamonds represent the fit of all quality factor values with (3), combining intrinsic and acoustic radiation loss based on the exact model developed by Wilson-Rae et al. [30]. The material properties of silicon were used for $E_{s}=130 \mathrm{GPa}$ and $\rho_{s}=2300 \mathrm{~kg} / \mathrm{m}^{3}$. $(\wedge:$ AND, $\vee:$ OR $)$. 
is accurately described by the intrinsic damping model (1) (red line). Hence, the maximal $Q$ s of the SR-SiN membrane seem to be clearly limited by intrinsic losses. In contrast, the peak $Q$ s of the high-stress membrane are below the intrinsic loss envelope and they thus seem to be limited by radiation loss. The combined model (3) is shown as diamonds. In both membranes, modes with $n \vee m \leq 2$ are suppressed strongest by acoustic radiation loss, as predicted by the model, and as it was suggested by [25]. Both Si chips were fixed to the piezoelectric actuator with a double sticky carbon tape. The resonance frequencies are in the $\mathrm{MHz}$ regime, which results in wavelengths in the Si that are larger than the Si chip thickness. Hence, the carbon tape and the piezoshaker become part of the substrate. The lower Young's modulus of the tape reduces the acoustic mismatch compared to a pure Si substrate, which is reflected in the fit parameter $\alpha=0.1<1$. The lower stress in the SR-SiN membrane results in a better acoustic mismatch $\eta$ and a lower $Q_{\text {intr }}$ envelope so that the maximal $Q$ s are limited by intrinsic losses, which entails $Q$ s that are less scattered compared to the high-stress membrane. SR-SiN membranes are thus the optimal structures to investigate the origin of the intrinsic loss, which is presented in the following part.

Figure 2(a) shows the extracted $Q_{\text {intr }}$ from the maximal $Q$ envelope given by intrinsic losses (1) from a set of square SR-SiN membranes with varying thicknesses $h$ and lengths $L$. The complete set of measured $Q_{\text {intr }}$ are plotted in the Supplemental Material [36]. The $Q_{\text {intr }}$ values increase steadily with membrane thickness $h$, independent of the membrane size $L$. For low $h$ the increase is following a linear trend (see linear slope line). A similar linear trend has been observed with Qs of SiN microcantilevers and was assigned to surface loss $Q_{\text {surf }}(h)=\beta h$, with a slope $\beta$ [37]. Hence, the observed linear relationship in Fig. 2(a) of $Q_{\text {intr }}$ with $h$ is strong evidence of surface loss. For structures with a reduced surface-to-volume ratio, surface loss will become obsolete and the intrinsic loss will be dominated by volume loss $Q_{\mathrm{vol}}$. This can be summarized by the formula

$$
Q_{\text {intr }}^{-1}(h)=Q_{\text {surf }}^{-1}(h)+Q_{\text {vol }}^{-1} .
$$

In order to get more data to test the model (4), we extract $Q_{\text {intr }}$ values for diverse $\mathrm{SiN}$ resonators from the literature. The values are obtained directly from maximal $Q$ s of unstressed cantilevers, and calculated by means of (1) from prestressed strings and membranes. All $Q_{\text {intr }}$ values are listed in Fig. 2(b) together with the average values from Fig. 2(a). All values are fitted with (4). Apparently, the trend of all $Q_{\text {intr }}$ s of all different $\mathrm{SiN}$ structures is described accurately by a combination of surface and volume loss. Our membranes had relatively large variations in $h, L$, and $\sigma$ of $\pm 15 \%, \pm 25 \%$, and $\pm 75 \%$, with respect to their nominal values, which propagates to a total uncertainty in the extraction of $Q_{\text {intr }}$ of $\pm 60 \%$. We took this as our error estimation for all values (thin red lines). From the fit, an
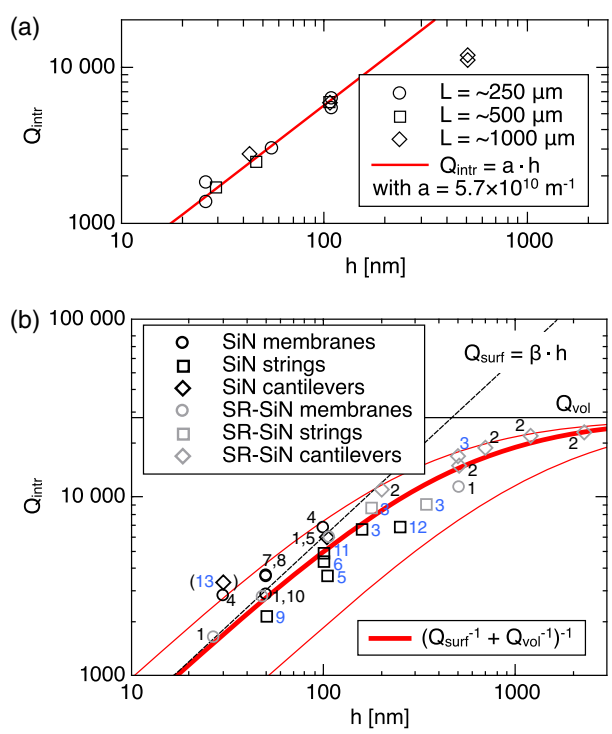

FIG. 2 (color online). (a) Intrinsic quality factors $Q_{\text {intr }}$ as a function of membrane thickness $h$ of SR-SiN membranes. The values represent the maximal values within the asymptotic $Q_{\text {intr }}$ envelope. The tensile stress varied strongly between $60 \mathrm{MPa}<$ $\sigma<253 \mathrm{MPa}$ (determined by means of resonance frequency). The lowest values are fitted with a linear slope. (b) $Q_{\text {intr }}$ values at room temperature extracted from literature and this work as a function of structure thickness $h$. The red line represents (4) fitted to all values with $\beta=6 \times 10^{10} \pm 4 \times 10^{10} \mathrm{~m}^{-1}$ and a volume loss related $Q_{\mathrm{vol}}=28000 \pm 2000$. The fine red lines represent the estimated error of $\beta$ of $\pm 60 \%$. The values are taken from 1: this work; 2: [37]; 3: [21]; 4: [24]; 5: [1]; 6: [22]; 7: [34]; 8: [7]; 9: [38]; 10: [25]; 11: [4]; 12: [39] (13: [40]— $h$ has a high uncertainty as it was only estimated from the HF etch rate; the initial thickness was $110 \mathrm{~nm})$. All values were extracted assuming $E=240 \mathrm{GPa}$ and $\rho=3000 \mathrm{~kg} / \mathrm{m}^{3}$. The blue numbered values represent $\mathrm{SiN}$ resonators that were in contact with hydrofluoric acid.

average surface loss parameter of $\beta=6 \times 10^{10} \pm 4 \times$ $10^{10} \mathrm{~m}^{-1}$ and a volume loss related $Q_{\mathrm{vol}}=28000 \pm$ 2000 can be extracted. It seems that all different structure types made from either SR-SiN or stoichiometric SiN are ultimately limited by surface loss. Volume loss starts to significantly contribute in thicker resonators.

The origin of the observed surface loss could be manifold, e.g., surface impurities or surface roughness. The chemical analysis with x-ray photoelectron spectroscopy of the surface of two SiN membranes [one commercial stoichiometric lowpressure chemical vapor deposition (LPCVD) SiN membrane from Norcada, and one stoichiometric LPCVD SiN membrane fabricated in-house] revealed a high concentration of oxygen and carbon (see Supplemental Material [36]). The same finding was made earlier by Yang et al. [41] who found oxygen and carbon concentration on the surface of LPCVD $\mathrm{SiN}$ of 22 and 10 at. \%, respectively. It has further been shown that these specific SiN surface impurities remain after cleaning with hydrofluoric acid (HF) and 3-7 at. \% of F impurities are added [42]. Hence, surface impurities seem to be ubiquitous in LPCVD SiN films and can even be increased, 
e.g., with an HF dip. In Fig. 2(b) all the SiN resonators that were exposed to HF are listed with blue numbers and their $Q_{\text {intr }}$ values are generally below the average. It seems that an $\mathrm{HF}$ dip could increase surface loss. Another possible origin of surface losses could be related to surface roughness which was found to be in the range of $0.3-3 \mathrm{~nm}[41,43]$ of untreated LPCVD SiN. Hence, surface roughness can become a significant fraction of the total SiN thin film thickness.

Based on the $Q_{\text {intr }}$ master curve for SiN from Fig. 2(b), it is now possible to predict the maximal obtainable $Q$ s for harmonic modes $n=m$ of square $\mathrm{SiN}$ membranes that are limited by intrinsic loss. From Fig. 3(a) it becomes evident that the thickness does not significantly influence $Q$ of thin membranes at low mode numbers, an effect that has been observed experimentally [23]. This is a direct effect of the $Q_{\text {intr }}$ that decreases with thickness and hence counteracts the $Q$-enhancing effect of a small $h$ in (1). Thinner membranes only result in higher $Q \mathrm{~s}$ at higher modes. For Fig. 3(b) the thickness is fixed to $30 \mathrm{~nm}$. It is not surprising that larger membranes result in higher $Q$ s. But $Q$ starts to deteriorate with mode numbers when $\lambda$ becomes large as, e.g., it was observed with short SiN strings [22]. In quantum cavity optomechanics a figure of merit is the $Q f$ product. $Q f>6 \times 10^{12} \mathrm{~Hz}$ is the minimum requirement for room-temperature quantum optomechanics [44]. In that case the thermal decoherence rate $\left(k_{B} T\right) /(2 \pi Q \hbar)$ at temperature $T$ is smaller than the resonance frequency $f$. The maximal $Q f$ product obtainable with a SiN membrane at room temperature is shown in Fig. 3(c). It seems that the limit cannot be overcome in the fundamental mode
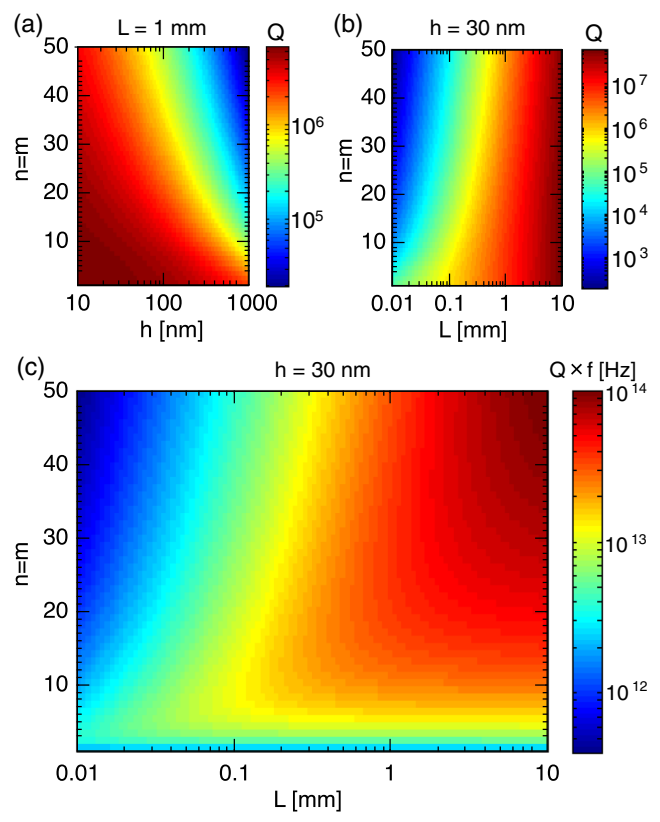

FIG. 3 (color online). Prediction of maximal $Q$ and $Q f$ values obtainable with a square SiN membrane at room temperature for harmonic modes $(n=m)$ that are limited by intrinsic loss. A value error of $60 \%$ has to be assumed. independent of membrane size, confirming the experimental findings from Wilson et al. [7]. For SiN string resonators, the maximal $Q$ values for low mode numbers are equal to the ones displayed in Figs. 3(a) and 3(b), but the $Q f$ product values have to be divided by $\sqrt{2}$. It has been shown that intrinsic damping is reduced at cryogenic temperatures, which means that all predicted values in Fig. 3 will increase accordingly $[34,40,45]$.

In conclusion, $Q$ in prestressed $\mathrm{SiN}$ micro- and nanomechanical resonators is limited by a combination of intrinsic and acoustic radiation loss. In membranes, both respective $Q$ s scale linearly with the dimensions $(L / h)$. Hence, the limiting damping mechanism is mainly determined by the acoustic mismatch of the membrane to the substrate $(\eta)$. In high-stress SiN membranes, $\eta$ is reduced and the maximal intrinsic loss $Q$ limit is increased; hence, the resulting $Q$ values are strongly mode dependent and are scattered due to radiation loss. The maximal $Q$ s can become limited by intrinsic loss by maximizing the acoustic mismatch, e.g., by mounting the chip freely or with a phononic band gap structure. In contrast, low-stress SiN membranes have a higher acoustic mismatch to the substrate and the maximal intrinsic $Q$ limit is lower. Hence, the resulting $Q$ s of higher mode numbers $(n \wedge m \geq 3)$ reach an upper envelope that is limited by intrinsic losses, while lower mode numbers $(n \vee m \leq 2)$ can be limited by radiation loss. Generally, radiation loss is minimal for symmetric modes $(n \sim m)$. In SiN strings, radiation loss scales inversely with width and narrow strings can become limited by intrinsic loss.

The intrinsic quality factors $Q_{\text {intr }}$ of thin low-stress SiN membranes scale linearly with the membrane thickness, which is strong evidence of surface loss. The same linear scaling of $Q_{\text {surf }}=\beta h$ has been confirmed by independent SiN $Q$ data taken from literature (cantilevers, strings and membranes) which is evidence that surface loss is the ubiquitous limiting damping mechanism in thin arbitrary $\mathrm{SiN}$ resonators with a scaling factor $\beta=6 \times 10^{10} \pm$ $4 \times 10^{10} \mathrm{~m}^{-1}$. For thin prestressed resonators that are limited by intrinsic loss, the thickness dependent surface loss is counteracting the $Q$ enhancement at low mode numbers and $Q$ can only significantly be increased with the size $L$. Finally, it seems that $Q f>6 \times 10^{12} \mathrm{~Hz}$ required for quantum cavity optomechanics at room temperature cannot be reached with the fundamental mode, independent of resonator length.

The framework of $Q$ analysis presented here is readily applicable to other emerging thin film mechanical string or membrane-type resonators made of materials such as $\mathrm{SiC}$ [46,47], InGaP [48], C (graphene) [49-51], C (diamond) [52], or $\mathrm{MoS}_{2}[53,54]$.

The authors would like to acknowledge A. Boisen for her support, and the staff in DTU-Danchip for help in the fabrication of the membranes. The authors further thank B. Amato for the help in the laboratory, A. Schliesser for his valuable input, and I. Wilson-Rae for the generous support with the radiation loss model. This research is supported 
by the VILLUM Young Investigator Programme (Project No. VKR023125) and the Swiss National Science Foundation (PP00P2-144695). The asymptotic limit of the radiation loss model was derived and generously provided by I. Wilson-Rae.

*sils@nanotech.dtu.dk

[1] S. S. Verbridge, J. M. Parpia, R. B. Reichenbach, L. M. Bellan, and H. G. Craighead, J. Appl. Phys. 99, 124304 (2006).

[2] B. M. Zwickl, W. E. Shanks, A. M. Jayich, C. Yang, B. Jayich, J. D. Thompson, and J. G. E. Harris, Appl. Phys. Lett. 92, 103125 (2008).

[3] J. D. Thompson, B. M. Zwickl, A. M. Jayich, F. Marquardt, S. M. Girvin, and J. G. E. Harris, Nature (London) 452, 72 (2008).

[4] E. Gavartin, P. Verlot, and T. J. Kippenberg, Nat. Nanotechnol. 7, 509 (2012).

[5] T. P. Purdy, R. W. Peterson, and C. A. Regal, Science 339, 801 (2013).

[6] G. A. Brawley, M. R. Vanner, P. E. Larsen, S. Schmid, A. Boisen, and W. P. Bowen, arXiv:1404.5746.

[7] D. J. Wilson, C. A. Regal, S. B. Papp, and H. J. Kimble, Phys. Rev. Lett. 103, 207204 (2009).

[8] T. Faust, P. Krenn, S. Manus, J. Kotthaus, and E. Weig, Nat. Commun. 3, 728 (2012).

[9] G. Anetsberger, E. Gavartin, O. Arcizet, Q.P. Unterreithmeier, E. M. Weig, M. L. Gorodetsky, J.P. Kotthaus, and T. J. Kippenberg, Phys. Rev. A 82, 061804 (2010).

[10] K. Hammerer, M. Wallquist, C. Genes, M. Ludwig, F. Marquardt, P. Treutlein, P. Zoller, J. Ye, and H. J. Kimble, Phys. Rev. Lett. 103, 063005 (2009).

[11] S. Camerer, M. Korppi, A. Jöckel, D. Hunger, T. W. Hänsch, and P. Treutlein, Phys. Rev. Lett. 107, 223001 (2011).

[12] R. W. Andrews, R. W. Peterson, T. P. Purdy, K. Cicak, R. W. Simmonds, C. A. Regal, and K. W. Lehnert, Nat. Phys. 10, 321 (2014).

[13] T. Bagci, A. Simonsen, S. Schmid, L. G. Villanueva, E. Zeuthen, J. Appel, J. M. Taylor, A. Sørensen, K. Usami, A. Schliesser, and E. S. Polzik, Nature (London) 507, 81 (2014).

[14] S. Schmid, T. Bagci, E. Zeuthen, J. M. Taylor, P. K. Herring, M. C. Cassidy, C. M. Marcus, L. Guillermo Villanueva, B. Amato, A. Boisen, Y. Cheol Shin, J. Kong, A. S. Sørensen, K. Usami, and E. S. Polzik, J. Appl. Phys. 115, 054513 (2014).

[15] M. S. Hanay, S. Kelber, A. K. Naik, D. Chi, S. Hentz, E. C. Bullard, E. Colinet, L. Duraffourg, and M. L. Roukes, Nat. Nanotechnol. 7, 602 (2012).

[16] R. A. Barton, B. Ilic, S. S. Verbridge, B. R. Cipriany, J. M. Parpia, and H. G. Craighead, Nano Lett. 10, 2058 (2010).

[17] S. Yamada, S. Schmid, T. Larsen, O. Hansen, and A. Boisen, Anal. Chem. 85, 10531 (2013).

[18] S. Schmid, M. Kurek, J. Q. Adolphsen, and A. Boisen, Sci. Rep. 3, 1288 (2013).

[19] T. Larsen, S. Schmid, L. G. Villanueva, and A. Boisen, ACS Nano 7, 6188 (2013).
[20] S. Schmid, K. Wu, P. E. Larsen, T. Rindzevicius, and A. Boisen, Nano Lett. 14, 2318 (2014).

[21] S. Schmid, K. D. Jensen, K. H. Nielsen, and A. Boisen, Phys. Rev. B 84, 165307 (2011).

[22] Q. P. Unterreithmeier, T. Faust, and J. P. Kotthaus, Phys. Rev. Lett. 105, 027205 (2010).

[23] V. P. Adiga, B. Ilic, R. A. Barton, I. Wilson-Rae, H. G. Craighead, and J. M. Parpia, J. Appl. Phys. 112, 064323 (2012).

[24] S. Chakram, Y. S. Patil, L. Chang, and M. Vengalattore, Phys. Rev. Lett. 112, 127201 (2014).

[25] P.-L. Yu, T. P. Purdy, and C. A. Regal, Phys. Rev. Lett. 108, 083603 (2012).

[26] G. I. Gonzfilez and P. R. Saulson, J. Acoust. Soc. Am. 96, 207 (1994).

[27] A. Boisen, S. Dohn, S. S. Keller, S. Schmid, and M. Tenje, Rep. Prog. Phys. 74, 036101 (2011).

[28] G. Cagnoli, J. Hough, D. Debra, M. M. Fejer, E. Gustafson, S. Rowan, and V. Mitrofanov, Phys. Lett. A 272, 39 (2000).

[29] I. Wilson-Rae, Phys. Rev. B 77, 245418 (2008).

[30] I. Wilson-Rae, R. A. Barton, S. S. Verbridge, D. R. Southworth, B. Ilic, H. G. Craighead, and J. M. Parpia, Phys. Rev. Lett. 106, 047205 (2011).

[31] M. C. Cross and R. Lifshitz, Phys. Rev. B 64, 085324 (2001).

[32] J. Rieger, A. Isacsson, M. J. Seitner, J. P. Kotthaus, and E. M. Weig, Nat. Commun. 5, 3345 (2014).

[33] D. J. Wilson, Ph.D. thesis, California Institute of Technology, 2012.

[34] Y. Tsaturyan, A. Barg, A. Simonsen, L. G. Villanueva, S. Schmid, A. Schliesser, and E. S. Polzik, Opt. Express 22, 6810 (2014).

[35] P.-L. Yu, K. Cicak, N. S. Kampel, Y. Tsaturyan, T. P. Purdy, R. W. Simmonds, and C. A. Regal, Appl. Phys. Lett. 104, 023510 (2014).

[36] See Supplemental Material at http://link.aps.org/ supplemental/10.1103/PhysRevLett.113.227201 for a model comparison of data from literature, the complete set of measured intrinsic $Q$ values, and the XPS spectra.

[37] K. Y. Yasumura, T. D. Stowe, E. M. Chow, T. Pfafman, T. W. Kenny, B. C. Stipe, and D. Rugar, J. Microelectromech. Syst. 9, 117 (2000).

[38] S. S. Verbridge, H. G. Craighead, and J. M. Parpia, Appl. Phys. Lett. 92, 013112 (2008).

[39] A. Suhel, B. D. Hauer, T. S. Biswas, K. S. D. Beach, and J. P. Davis, Appl. Phys. Lett. 100, 173111 (2012).

[40] D. R. Southworth, R. A. Barton, S. S. Verbridge, B. Ilic, A. D. Fefferman, H. G. Craighead, and J. M. Parpia, Phys. Rev. Lett. 102, 225503 (2009).

[41] G.-R. Yang, Y.-P. Zhao, Y. Hu, T. Paul Chow, and R. J. Gutmann, Thin Solid Films 333, 219 (1998).

[42] P. J. French, P. M. Sarro, R. Malle, E. J. M. Fakkeldij, and R. F. Wolffenbuttel, Sens. Actuators A 58, 149 (1997).

[43] C. Gui, H. Albers, J. G. E. Gardeniers, M. Elwenspoek, and P. V. Lambeck, Microsyst. Technol. 3, 122 (1997).

[44] M. Aspelmeyer, T. J. Kippenberg, and F. Marquardt, arXiv:1303.0733.

[45] T. Faust, J. Rieger, M. J. Seitner, J. P. Kotthaus, and E. M. Weig, Phys. Rev. B 89, 100102 (2014).

[46] X. L. Feng, C. J. White, A. Hajimiri, and M. L. Roukes, Nat. Nanotechnol. 3, 342 (2008). 
[47] I. Bargatin, I. Kozinsky, and M. L. Roukes, Appl. Phys. Lett. 90, 093116 (2007).

[48] G. D. Cole, P.-L. Yu, C. Gärtner, K. Siquans, R. Moghadas Nia, J. Schmöle, J. Hoelscher-Obermaier, T. P. Purdy, W. Wieczorek, C. A. Regal, and M. Aspelmeyer, Appl. Phys. Lett. 104, 201908 (2014).

[49] R. A. Barton, I. R. Storch, V. P. Adiga, R. Sakakibara, B. R. Cipriany, B. Ilic, S. P. Wang, P. Ong, P. L. McEuen, J. M. Parpia, and H. G. Craighead, Nano Lett. 12, 4681 (2012).

[50] P. Weber, J. Güttinger, I. Tsioutsios, D. E. Chang, and A. Bachtold, Nano Lett. 14, 2854 (2014).
[51] X. Song, M. Oksanen, J. Li, P. J. Hakonen, and M. A. Sillanpää, Phys. Rev. Lett. 113, 027404 (2014).

[52] A. C. Barnes and C. A. Zorman, in Proceedings of the 2013 IEEE Sensors Conference (IEEE, New York, 2013), pp. 1-4.

[53] J. Lee, Z. Wang, K. He, J. Shan, and P. X.-L. Feng, ACS Nano 7, 6086 (2013).

[54] A. Castellanos-Gomez, R. van Leeuwen, M. Buscema, H. S. J. van der Zant, G. A. Steele, and W. J. Venstra, Adv. Mater. 25, 6719 (2013). 